\title{
Parameter-dependent Stokes problems in vector-valued spaces and applications
}

\author{
Veli B Shakhmurov*
}

Dedicated to International Conference on the Theory, Methods and Applications of Nonlinear Equations in Kingsville, TX-USA, Texas A\&M University-Kingsville-2012

\section{"Correspondence:}

veli.sahmurov@okan.edu.tr Department of Mechanical Engineering, Okan University, Akfirat, Tuzla, Istanbul 34959, Turkey Institute of Mathematics and Mechanics, Azerbaijan National Academy of Sciences, Baku, Azerbaijan

\begin{abstract}
The stationary and instationary Stokes equations with operator coefficients in abstract function spaces are studied. The problems are considered in the whole space, and equations include small parameters. The uniform separability of these problems is established.

MSC: 35Q30; 76D05; 34G10; 35J25

Keywords: Stokes systems; Navier-Stokes equations; differential equations with small parameters; semigroups of operators; boundary value problems; differential-operator equations; maximal $L^{p}$ regularity
\end{abstract}

\section{Introduction}

We consider the initial value problem (IVP) for the following Stokes equation with small parameter:

$$
\begin{aligned}
& \frac{\partial u}{\partial t}-\Delta_{\varepsilon} u+A u+\nabla \varphi=f(x, t), \quad x \in R^{n}, t \in(0, T), \\
& \operatorname{div} u=0, \quad u(x, 0)=a(x),
\end{aligned}
$$

where $\Delta_{\varepsilon} u=\sum_{k=1}^{n} \varepsilon_{k} \frac{\partial^{2} u}{\partial x_{k}^{2}}, A$ is a linear operator in a Banach space $E$ and $\varepsilon_{k}$ are a small positive parameters. Here $u=\left(u_{1}(x, t), u_{2}(x, t), \ldots, u_{n}(x, t)\right), \varphi=\varphi(x, t)$ are $E$-valued unknown solutions, $f=\left(f_{1}(x, t), f_{2}(x, t), \ldots, f_{n}(x, t)\right)$ is a given function and $a=\left(a_{1}(x), a_{2}(x), \ldots, a_{n}(x)\right)$ is an initial date. This problem is characterized by the presence of an abstract operator $A$ and small terms $\varepsilon_{k}$ which correspond to the inverse of Reynolds number Re very large. We prove that problem (1.1)-(1.2) has a unique strong maximal regular solution $u$ on a time interval $[0, T]$ independent of $\varepsilon_{k}$. For $\varepsilon_{k}=1, E=\mathbb{C}, A=b$, problem (1.1)-(1.2) is reduced to the Stokes problem

$$
\begin{aligned}
& \frac{\partial u}{\partial t}-\Delta u+b u+\nabla \varphi=f(x, t), \quad \operatorname{div} u=0, \\
& u(x, 0)=a(x), \quad x \in R^{n}, t \in(0, T),
\end{aligned}
$$

where $\mathbb{C}$ is the set of complex numbers and $b$ is a positive constant.

\section{盟 Springer}

C 2013 Shakhmurov; licensee Springer. This is an Open Access article distributed under the terms of the Creative Commons Attribution License (http://creativecommons.org/licenses/by/2.0), which permits unrestricted use, distribution, and reproduction in any medium, provided the original work is properly cited. 
Note that the existence of weak or strong solutions and regularity properties for the classical Stokes problems has been extensively studied, e.g., in [1-10]. There is an extensive literature on the solvability of the IVPs for the Stokes equation (see, e.g., $[1,3,10]$ and further papers cited there). Solonnikov [8] proved that for every $f \in L^{p}\left(\Omega \times(0, T) ; R^{3}\right)=$ $B(p), p \in(1, \infty)$, the time-dependent Stokes problem

$$
\begin{aligned}
& \frac{\partial u}{\partial t}-\Delta u+\nabla \varphi=f(x, t), \quad \operatorname{div} u=0,\left.\quad u\right|_{\partial \Omega}=0, \\
& u(x, 0)=0, \quad x \in \Omega, t \in(0, T)
\end{aligned}
$$

has a unique solution $(u, \nabla \varphi)$ so that

$$
\left\|\frac{\partial u}{\partial t}\right\|_{B(p)}+\left\|\nabla^{2} u\right\|_{B(p)}+\|\nabla \varphi\|_{B(p, q)} \leq C\|f\|_{B(p, q)}
$$

Then Giga and Sohr [3] improved the result of Solonnikov for spaces with different exponents in space and time, i.e., they proved that for $f \in L^{p}\left(0, T ;\left(L^{q}(\Omega)\right)^{n}\right)=B(p, q)$ there is a unique solution $(u, \nabla \varphi)$ of problem (1.5) so that

$$
\left\|\frac{\partial u}{\partial t}\right\|_{B(p, q)}+\left\|\nabla^{2} u\right\|_{B(p, q)}+\|\nabla \varphi\|_{B(p, q)} \leq C\|f\|_{B(p, q), \quad p, q \in(1, \infty) .}
$$

Moreover, the estimate obtained was global in time, i.e., the constant $C=C(\Omega, p, q)$ is independent of $T$ and $f$. To derive global $L^{p}-L^{q}$ estimates (1.6), Giga and Sohr used the abstract parabolic semigroup theory in $U M D$-type Banach spaces. Estimate (1.6) allows to study the existence of a solution and regularity properties of the corresponding NavierStokes problem (see, e.g., [5]).

In this paper, first we consider the following differential operator equation (DOE) with small parameters:

$$
-\Delta_{\varepsilon} u+(A+\lambda) u=f(x), \quad x \in R^{n},
$$

where $A$ is a linear operator in a Banach space $E, \varepsilon_{k}$ are positive and $\lambda$ is a complex parameter.

We show that for $f \in W^{m, q}\left(R^{n} ; E\right), \lambda \in S_{\psi}$, problem (1.7) has a unique solution $u$ belonging to $W^{2+m, q}\left(R^{n} ; E(A), E\right)$ and the uniform coercive estimate holds

$$
\sum_{k=1}^{n} \sum_{i=0}^{m+2} \varepsilon_{k}^{\frac{i}{m+2}}|\lambda|^{1-\frac{i}{m+2}}\left\|\frac{\partial^{i} u}{\partial x_{k}^{i}}\right\|_{L^{q}\left(R^{n} ; E\right)}+\|A u\|_{L^{q}\left(R^{n} ; E\right)} \leq C\|f\|_{W^{m, q}\left(R^{n} ; E\right)},
$$

where $C(q)$ is independent of $\varepsilon_{1}, \varepsilon_{2}, \ldots, \varepsilon_{n}, \lambda$ and $f$.

We consider, then, the stationary abstract Stokes problem with small parameters

$$
-\Delta_{\varepsilon} u+A u+\nabla \varphi=f(x), \quad \operatorname{div} u=0, \quad x \in R^{n},
$$

where $f=\left(f_{1}(x), f_{2}(x), \ldots, f_{n}(x)\right)$ is data and $u=\left(u_{1}(x), u_{2}(x), \ldots, u_{n}(x)\right)$ is a solution. By applying the projection transformation $P$, equation (1.8) can be reduced to the following 
problem:

$$
-P \Delta_{\varepsilon} u+A u=f(x), \quad x \in R^{n} .
$$

Let $O_{\varepsilon, q}$ denote the operator generated by problem (1.9), i.e., $O_{\varepsilon, q}$ is a Stokes operator in solenoidal space $L_{\sigma}^{q}\left(R^{n} ; E\right)$ defined by

$$
\begin{aligned}
& D\left(O_{\varepsilon, q}\right)=\left(W_{\sigma}^{2, q}\left(R^{n} ; E(A), E\right)\right)^{n}=\left\{u \in\left(W^{2, q}\left(R^{n} ; E(A), E\right)\right)^{n}, \operatorname{div} u=0\right\}, \\
& O_{\varepsilon, q} u=-P \Delta_{\varepsilon} u+A u .
\end{aligned}
$$

We prove that the operator $O_{\varepsilon, q}$ is uniformly positive and also is a generator of an analytic semigroup in $L_{\sigma}^{q}\left(R^{n} ; E\right)$. Finally, the instationary Stokes problem (1.1)-(1.3) is considered and the well-posedness of this problem is derived. In application we show the separability properties of the anisotropic stationary Stokes operator in mixed $L^{\mathbf{p}}$ spaces and maximal regularity properties of infinity system of instationary Stokes equations in $L^{p}$ spaces.

\section{Notations and background}

Let $E$ be a Banach space and let $L^{p}(\Omega ; E)$ denote the space of strongly measurable $E$-valued functions that are defined on the measurable subset $\Omega \subset R^{n}$ with the norm

$$
\|f\|_{L^{p}}=\|f\|_{L^{p}(\Omega ; E)}=\left(\int_{\Omega}\|f(x)\|_{E}^{p} d x\right)^{\frac{1}{p}}, \quad 1 \leq p<\infty
$$

The Banach space $E$ is called a $U M D$-space if the Hilbert operator

$$
(H f)(x)=\lim _{\varepsilon \rightarrow 0} \int_{|x-y|>\varepsilon} \frac{f(y)}{x-y} d y
$$

is bounded in $L_{p}(R, E), p \in(1, \infty)$ (see, e.g., [11]). UMD spaces include, e.g., $L_{p}, l_{p}$ spaces and Lorentz spaces $L_{p q}, p, q \in(1, \infty)$.

Let $E_{1}$ and $E_{2}$ be two Banach spaces. $B\left(E_{1}, E_{2}\right)$ denotes the space of bounded linear operators from $E_{1}$ into $E_{2}$ endowed with the usual uniform operator topology. For $E_{1}=E_{2}=E$, it is denoted by $B(E)$. Now $\left(E_{1}, E_{2}\right)_{\theta, p}, 0<\theta<1,1 \leq p \leq \infty$, denotes interpolation spaces defined by the $K$ method [12, $\$ 1.3 .1$.

Let

$$
S_{\psi}=\{\lambda \in \mathbb{C},|\arg \lambda| \leq \varphi \cup\{0\}, 0 \leq \psi<\pi\} .
$$

A linear operator $A$ is said to be $\psi$-positive in a Banach space $E$ with bound $M>0$ if the domain $D(A)$ is dense on $E$ and $\left\|(A+\lambda I)^{-1}\right\|_{B(E)} \leq M(1+|\lambda|)^{-1}$ for any $\lambda \in S_{\psi}, 0 \leq \psi<\pi$, where $I$ is the identity operator in $E$. It is known $[12, \$ 1.15 .1]$ that there exist the fractional powers $A^{\theta}$ of the positive operator $A$. Let $E\left(A^{\theta}\right)$ denote the space $D\left(A^{\theta}\right)$ with the norm

$$
\|u\|_{E\left(A^{\theta}\right)}=\left(\|u\|^{p}+\left\|A^{\theta} u\right\|^{p}\right)^{\frac{1}{p}}, \quad 1 \leq p<\infty, 0<\theta<\infty .
$$

$\mathbb{N}$ denotes the set of natural numbers. A set $G \subset B\left(E_{1}, E_{2}\right)$ is called $R$-bounded (see, e.g., [11]) if there is a positive constant $C$ such that for all $T_{1}, T_{2}, \ldots, T_{m} \in G$ and $u_{1}, u_{2}, \ldots, u_{m} \in$ 
$E_{1}, m \in \mathbb{N}$

$$
\int_{\Omega}\left\|\sum_{j=1}^{m} r_{j}(y) T_{j} u_{j}\right\|_{E_{2}} d y \leq C \int_{\Omega}\left\|\sum_{j=1}^{m} r_{j}(y) u_{j}\right\|_{E_{1}} d y,
$$

where $\left\{r_{j}\right\}$ is a sequence of independent symmetric $\{-1,1\}$-valued random variables on $\Omega$. The smallest $C$, for which the estimate above holds, is called an $R$-bound of the collection $G$ and denoted by $R(G)$.

A set $G_{h} \subset B\left(E_{1}, E_{2}\right)$ depending of parameter $h \in Q$ is called uniform $R$-bounded with respect to $h$ if there is a constant $C$, independent of $h \in Q$ such that for all $T_{1}(h), T_{2}(h), \ldots, T_{m}(h) \in G_{h}$ and $u_{1}, u_{2}, \ldots, u_{m} \in E_{1}, m \in \mathbb{N}$,

$$
\int_{\Omega}\left\|\sum_{j=1}^{m} r_{j}(y) T_{j}(h) u_{j}\right\|_{E_{2}} d y \leq C \int_{\Omega}\left\|\sum_{j=1}^{m} r_{j}(y) u_{j}\right\|_{E_{1}} d y .
$$

It implies that $\sup _{h \in Q} R\left(G_{h}\right) \leq C$.

The $\psi$-positive operator $A$ is said to be $R$-positive in a Banach space $E$ if the set $L_{A}=$ $\left\{\xi(A+\xi)^{-1}: \xi \in S_{\psi}\right\}, 0 \leq \psi<\pi$, is $R$-bounded.

The operator $A(t)$ is said to be $\psi$-positive in $E$ uniformly with respect to $t$ with bound $M>0$ if $D(A(t))$ is independent of $t, D(A(t))$ is dense in $E$ and $\left\|(A(t)+\lambda)^{-1}\right\| \leq M(1+|\lambda|)^{-1}$ for all $\lambda \in S_{\psi}, 0 \leq \psi<\pi$, where $M$ does not depend of $t$ and $\lambda$.

Let $E_{0}$ and $E$ be two Banach spaces and let $E_{0}$ be continuously and densely embedded into $E$. Let $\Omega$ be a measurable set in $R^{n}$ and $m$ be a positive integer. $W^{p, m}\left(\Omega ; E_{0}, E\right)$ denotes the class of all functions $u \in L^{p}\left(\Omega ; E_{0}\right)$ that have the generalized derivatives $\frac{\partial^{m} u}{\partial x_{k}^{m}} \in L^{p}(\Omega ; E)$ with the norm

$$
\|u\|_{W^{p}, m\left(\Omega ; E_{0}, E\right)}=\|u\|_{L^{p}\left(\Omega ; E_{0}\right)}+\sum_{k=1}^{n}\left\|\frac{\partial^{m} u}{\partial x_{k}^{m}}\right\|_{L^{p}(\Omega ; E)}<\infty .
$$

For $n=1, \Omega=(a, b), a, b \in \mathbb{R}$, the space $W^{p, m}\left(\Omega ; E_{0}, E\right)$ is denoted by $W^{p, m}\left(a, b ; E_{0}, E\right)$. For $E_{0}=E$ the space $W^{p, m}\left(\Omega ; E_{0}, E\right)$ is denoted by $W^{p, m}(\Omega ; E)$.

Let $H^{q, s}\left(R^{n} ; E\right),-\infty<s<\infty$ denote an $E$-valued Liouville space of order $s$, i.e.,

$$
H^{q, s}\left(R^{n} ; E\right)=\left\{u \in L^{q}\left(R^{n} ; E\right),\|u\|_{H^{q, s}\left(R^{n} ; E\right)}=\left\|F^{-1}\left(1+|\xi|^{2}\right)^{\frac{s}{2}} F u\right\|_{L^{q}\left(R^{n} ; E\right)}<\infty\right\},
$$

where $F$ and $F^{-1}$ denote the Fourier and inverse Fourier transforms, respectively.

Let $H^{q, s}\left(R^{n} ; E_{0}, E\right)$ be a Liouville-Lions type space, i.e.,

$$
\begin{aligned}
H^{q, s}\left(R^{n} ; E_{0}, E\right)= & \left\{u \in H^{q, s}\left(R^{n} ; E\right) \cap L^{q}\left(R^{n} ; E_{0}\right),\right. \\
& \left.\|u\|_{H^{q, s}\left(R^{n} ; E_{0}, E\right)}=\|u\|_{L^{q}\left(R^{n} ; E_{0}\right)}+\|u\|_{H^{q, s}\left(R^{n} ; E\right)}<\infty\right\} .
\end{aligned}
$$

For $\varepsilon=\left(\varepsilon_{1}, \varepsilon_{2}, \ldots, \varepsilon_{n}\right)$ we define the parameter-dependent norm in $H^{q, s}\left(R^{n} ; E_{0}, E\right)$ such that

$$
\|u\|_{H_{\varepsilon}^{q, s}\left(R^{n} ; E_{0}, E\right)}=\|u\|_{L^{q\left(R^{n} ; E_{0}\right)}}+\left\|F^{-1}\left(1+\sum_{k=1}^{n} \varepsilon_{k} \xi_{k}^{2}\right)^{\frac{s}{2}} F u\right\|_{L^{q}\left(R^{n} ; E\right)}<\infty
$$


Sometimes we use one and the same symbol $C$ without distinction to denote positive constants which may differ from each other even in a single context. When we want to specify the dependence of such a constant on a parameter, say $\alpha$, we write $C_{\alpha}$.

\section{Boundary value problems for abstract elliptic equations}

In this section, we derive the maximal regularity properties of problem (1.7).

BVPs for DOEs were studied, e.g., in [9, 11, 13-19]. For references, see, e.g., [19]. From [18, Theorem 4.1] we have the following result.

Theorem 3.1 Let E be a UMD space and let $A$ be an R-positive operator in $E$ for $0 \leq \psi<\pi$. Then problem (1.7) has a unique solution $u \in W^{q, 2}\left(R^{n} ; E(A), E\right)$ for $f \in L^{q}\left(R^{n} ; E\right)$ and $\lambda \in S_{\psi}$. Moreover, the following uniform coercive estimate holds:

$$
\sum_{k=1}^{n} \sum_{i=0}^{2} \varepsilon_{k}^{\frac{i}{2}}|\lambda|^{1-\frac{i}{2}}\left\|\frac{\partial^{i} u}{\partial x_{k}^{i}}\right\|_{L^{q}\left(R^{n} ; E\right)}+\|A u\|_{L^{q}(G ; E)} \leq C\|f\|_{L^{q}\left(R^{n} ; E\right)}
$$

with $C(q)$ independent of $\varepsilon_{1}, \varepsilon_{2}, \ldots, \varepsilon_{n}, \lambda$ and $f$.

Consider the differential operator $Q_{\varepsilon}=Q_{\varepsilon q}$ in $L^{q}\left(R^{n} ; E\right)$ generated by problem (3.1), i.e.,

$$
D\left(Q_{\varepsilon}\right)=W^{q, 2}\left(R^{n}\right), \quad Q_{\varepsilon} u=-\Delta_{\varepsilon} u+A u .
$$

Let $B_{q}=B\left(L^{q}\left(R^{n} ; E\right)\right)$. From Theorem 3.1 we obtain the following.

Result 3.1 For $\lambda \in S_{\psi}$, there is a resolvent $\left(Q_{\varepsilon}+\lambda\right)^{-1}$ satisfying the uniform estimate

$$
\sum_{k=1}^{n} \sum_{i=0}^{2}|\lambda|^{1-\frac{i}{2}} \varepsilon_{k}^{\frac{i}{2}}\left\|\frac{\partial^{i}}{\partial x_{k}^{i}}\left(Q_{\varepsilon}+\lambda\right)^{-1}\right\|_{B_{q}}+\left\|A\left(Q_{\varepsilon}+\lambda\right)^{-1}\right\|_{B_{q}} \leq C .
$$

Next we show the smoothness of problem (3.1). The main result is the following.

Theorem 3.2 Assume that $E$ is a UMD space, $A$ is an R-positive operator in $E, q \in(1, \infty)$ and $m$ is a positive integer.

Then, for all $f \in W^{q, m}\left(R^{n} ; E\right), \lambda \in S_{\psi}$, problem (3.1) has a unique solution $u$ that belongs to $W^{q, 2+m}\left(R^{n} ; E(A), E\right)$ and the following uniform coercive estimate holds:

$$
\sum_{k=1}^{n} \sum_{i=0}^{m+2} \varepsilon_{k}^{\frac{i}{m+2}}|\lambda|^{1-\frac{i}{m+2}}\left\|\frac{\partial^{i} u}{\partial x_{k}^{i}}\right\|_{W^{q, m}\left(R^{n} ; E\right)}+\|A u\|_{L^{q}\left(R^{n} ; E\right)} \leq C\|f\|_{W^{q, m}\left(R^{n} ; E\right)}
$$

with $C=C(q, A)$ independent of $\varepsilon_{1}, \varepsilon_{2}, \ldots, \varepsilon_{n}, \lambda$ and $f$.

Proof A solution of equation (1.7) is given by

$$
u(x)=F^{-1} L^{-1}(\lambda, \varepsilon, \xi) F f=\frac{1}{(2 \pi)^{n / 2}} \int_{R^{n}} e^{i \xi x} L^{-1}(\lambda, \varepsilon, \xi) \hat{f}(\xi) d \xi
$$


where $L(\lambda, \varepsilon, \xi)=A+\sum_{k=1}^{n} \varepsilon_{k} \xi_{k}^{2}+\lambda$ and $\hat{f}(\xi)=F f$. It follows from the expression above that

$$
\begin{aligned}
\sum_{k=1}^{n} & \sum_{i=0}^{m+2} \varepsilon_{k}^{\frac{i}{m+2}}|\lambda|^{1-\frac{i}{m+2}}\left\|\frac{\partial^{i} u}{\partial x_{k}^{i}}\right\|_{L^{q}\left(R^{n} ; E\right)}+\|A u\|_{L^{q}\left(R^{n} ; E\right)} \\
= & \sum_{k=1}^{n} \sum_{i=0}^{m+2} \varepsilon_{k}^{\frac{i}{m+2}}|\lambda|^{1-\frac{i}{m+2}}\left\|F^{-1} \xi_{k}^{i} L^{-1}(\lambda, \varepsilon, \xi) \hat{f}\right\|_{L^{q}\left(R^{n} ; E\right)} \\
& +\left\|F^{-1} A L^{-1}(\lambda, \varepsilon, \xi) \hat{f}\right\|_{L^{q}\left(R^{n} ; E\right)^{.}}
\end{aligned}
$$

It is sufficient to show that the operator-functions

$$
\begin{aligned}
& \Psi_{\xi \lambda}(\xi)=A L^{-1}(\lambda, \varepsilon, \xi)\left(1+\sum_{k=1}^{n}\left|\xi_{k}\right|^{m}\right)^{-1}, \\
& \sigma_{\varepsilon \lambda}(\xi)=\sum_{k=1}^{n} \sum_{i=0}^{m+2} \varepsilon_{k}^{\frac{i}{m+2}}|\lambda|^{1-\frac{i}{m+2}} \xi_{k}^{i}\left(1+\sum_{k=1}^{n}\left|\xi_{k}\right|^{m}\right)^{-1} L^{-1}(\lambda, \varepsilon, \xi)
\end{aligned}
$$

are uniform Fourier multipliers in $L^{q}\left(R^{n} ; E\right)$. Actually, due to positivity of $A$, we have

$$
\begin{aligned}
& \left\|L^{-1}(\lambda, \varepsilon, \xi)\right\| \leq M\left(1+\sum_{k=1}^{n} \varepsilon_{k} \xi_{k}^{2}+|\lambda|\right)^{-1} \\
& \left\|\Psi_{\varepsilon, \lambda}(\xi)\right\|=\left\|A L^{-1}(\lambda, \varepsilon, \xi)\right\|\left(1+\sum_{k=1}^{n}\left|\xi_{k}\right|^{m}\right)^{-1} \leq C\left(1+\sum_{k=1}^{n}\left|\xi_{k}\right|^{m}\right)^{-1} .
\end{aligned}
$$

It is clear to observe that

$$
\begin{aligned}
\xi_{k} \frac{\partial}{\partial \xi_{k}} \Psi_{\varepsilon \lambda}(\xi) & =-2 \varepsilon_{k} \xi_{k}^{2} A L^{-2}(\lambda, \varepsilon, \xi) \\
& =\left[-2 \varepsilon_{k} \xi_{k}^{2} L^{-1}(\lambda, \varepsilon, \xi)\right] A L^{-1}(\lambda, \varepsilon, \xi), \quad k=1,2, \ldots, n .
\end{aligned}
$$

Due to $R$-positivity of the operator $A$, the sets

$$
\left\{\left[-2 \varepsilon_{k} \xi_{k}^{2} L^{-1}(\lambda, \varepsilon, \xi)\right]: \xi \in R^{n} \backslash\{0\}\right\}, \quad\left\{A L^{-1}(\lambda, \varepsilon, \xi): \xi \in R^{n} \backslash\{0\}\right\}
$$

are $R$-bounded. So, in view of Kahane's contraction principle and from the product properties of the collection of $R$-bounded operators (see, e.g., [11], Lemma 3.5, Proposition 3.4), we obtain

$$
R\left\{\xi_{k} \frac{\partial}{\partial \xi_{k}} \Psi_{\varepsilon \lambda}(\xi): \xi \in R^{n} \backslash\{0\}\right\} \leq C_{k} .
$$

Namely, the $R$-bounds of sets $\left\{\xi_{k} \frac{\partial}{\partial \xi_{k}} \Psi_{\varepsilon \lambda}(\xi): \xi \in R^{n} \backslash\{0\}\right\}$ are independent of $\varepsilon$ and $\lambda$. Next, let us consider $\sigma_{\varepsilon \lambda}(\xi)$. It is clear to see that

$$
\left\|\sigma_{\varepsilon \lambda}(\xi)\right\|_{B(E)} \leq C|\lambda| \sum_{k=1}^{n} \sum_{i=0}^{m+2}\left[\varepsilon_{k}^{\frac{1}{m+2}}\left|\xi_{k}\right||\lambda|^{-\frac{1}{m+2}}\right]^{i}\left(1+\sum_{k=1}^{n}\left|\xi_{k}\right|^{m}\right)^{-1}\left\|L^{-1}(\lambda, \varepsilon, \xi)\right\|_{B(E)} .
$$


By using the well-known inequality

$$
\prod_{k=1}^{n} y_{k}^{\alpha_{k}} \leq C\left(1+\sum_{k=1}^{n} y_{k}^{l}\right), \quad \alpha_{k}, y_{k} \geq 0,|\alpha| \leq l
$$

for $y_{k}=\left(\varepsilon_{k}^{\frac{1}{2}}|\lambda|^{-\frac{1}{2}}\left|\xi_{k}\right|\right)$ and $l=m+2$, we get the uniform estimate

$$
\left|\sum_{k=1}^{n} \sum_{i=0}^{m+2}\left[\varepsilon_{k}^{\frac{i}{m+2}}\left|\xi_{k}\right||\lambda|^{-\frac{i}{m+2}}\right]\right| \leq C|\lambda|\left(1+\sum_{k=1}^{n}\left|\xi_{k}\right|^{m}\right)\left(1+|\lambda|^{-1} \sum_{k=1}^{n} \varepsilon_{k}\left|\xi_{k}\right|^{m+2}\right)^{-1} .
$$

From (3.4) and (3.5) we have the uniform estimate

$$
\left\|\sigma_{\varepsilon \lambda}(\xi)\right\|_{B(E)} \leq C|\lambda|\left(1+|\lambda|^{-1} \sum_{k=1}^{n} \varepsilon_{k}\left|\xi_{k}\right|^{m+2}\right)\left(1+\sum_{k=1}^{n}\left|\xi_{k}\right|^{m}\right)^{-1}\left\|L^{-1}(\lambda, \varepsilon, \xi)\right\| \leq C
$$

Due to $R$-positivity of the operator $A$, the set

$$
\left\{\left(|\lambda|+\sum_{k=1}^{n} \varepsilon_{k} \xi_{k}^{2}\right) L^{-1}(\lambda, \varepsilon, \xi): \xi \in R^{n} \backslash\{0\}\right\}
$$

is $R$-bounded. By using this fact, in view of (3.4) and Kahane's contraction principle, we obtain the $R$-boundedness of the set $\left\{\sigma_{\varepsilon \lambda}(\xi): \xi \in R \backslash\{0\}\right\}$. In a similar way, we obtain the uniform estimates

$$
\left\|\frac{\partial}{\partial \xi_{k}} \Psi_{\varepsilon, \lambda}(\xi)\right\|_{B(E)} \leq C_{1}, \quad\left\|\frac{\partial}{\partial \xi_{k}} \sigma_{\varepsilon \lambda}(\xi)\right\|_{B(E)} \leq C_{2}
$$

Let

$$
\begin{aligned}
& \sigma_{k \varepsilon \lambda}(\xi)=\left\{\xi_{k} \frac{\partial}{\partial \xi_{k}} \sigma_{\varepsilon \lambda}(\xi): \xi \in R^{n} \backslash\{0\}\right\}, \\
& \Psi_{k \varepsilon \lambda}(\xi)=\left\{\xi_{k} \frac{\partial}{\partial \xi_{k}} \Psi_{\varepsilon \lambda}(\xi): \xi \in R^{n} \backslash\{0\}\right\} .
\end{aligned}
$$

By the aid of the estimates above, due to $R$-positivity of the operator $A$, in view of estimate (3.4), by virtue of Kahane's contraction principle, from the additional and product properties of the collection of $R$-bounded operators, for $\xi_{1}, \xi_{2}, \ldots, \xi_{\mu} \in R, u_{1}, u_{2}, \ldots, u_{\mu} \in E$ and independent symmetric $\{-1,1\}$-valued random variables $r_{j}(y), j=1,2, \ldots, \mu, \mu \in \mathbb{N}$, we obtain the uniform estimate

$$
\begin{aligned}
& \int_{\Omega}\left\|\sum_{j=1}^{\mu} r_{j}(y) \sigma_{k \varepsilon \lambda}\left(\xi^{(j)}\right) u_{j}\right\|_{E} d y \\
& \quad \leq C \sum_{k=1}^{n} \int_{\Omega}\left\|\sum_{j=1}^{\mu} \sigma_{k \varepsilon \lambda}\left(\xi^{(j)}\right) r_{j}(y) u_{j}\right\|_{E} d y \\
& \quad \leq C \sup _{\varepsilon, \lambda} R\left(\left\{\xi_{k} \frac{\partial}{\partial \xi} \sigma_{k \varepsilon \lambda}(\xi): \xi \in R \backslash\{0\}\right\}\right) \int_{\Omega}\left\|\sum_{j=1}^{\mu} r_{j}(y) u_{j}\right\|_{E} d y \leq C_{k} .
\end{aligned}
$$


The same estimates are obtained for $\Psi_{k \varepsilon \lambda}(\xi)$ in a similar way. Hence, by virtue of [11, Theorem 3.4] it follows that $\Psi_{\varepsilon, \lambda}(\xi)$ and $\sigma_{\varepsilon \lambda}(\xi)$ are the uniform collection of multipliers in $L^{p}\left(R^{n} ; E\right)$. Then, by using equality (3.3), we obtain the assertion.

\section{The stationary Stokes system with small parameters}

In this section we derive the maximal regularity properties of the stationary abstract Stokes problem (1.8).

Let $H^{q, s}\left(R^{n} ; E\right),-\infty<s<\infty$ denote the $E$-valued Liouville space of order $s$ such that $H^{q, 0}\left(R^{n} ; E\right)=L^{q}\left(R^{n} ; E\right)$. It is known that if $E$ is a $U M D$ space, then $H^{q, m}\left(R^{n} ; E\right)=W^{q, m}\left(R^{n} ; E\right)$ for a positive integer $m$ (see, e.g., $[20, \mathbb{\$ 1 5}])$. For $q \in(1, \infty)$ let $X_{q}=\left(L^{q}\left(R^{n} ; E\right)\right)^{n}$ denote the space of an $E$-valued system of functions $f=\left(f_{1}(x), f_{2}(x), \ldots, f_{n}(x)\right)$ with the norm

$$
\|f\|_{X_{q}}=\left(\sum_{i=1}^{n}\left\|f_{i}\right\|_{L^{q}\left(R^{n} ; E\right)}^{q}\right)^{\frac{1}{q}} .
$$

$X_{q \sigma}=L_{\sigma}^{q}\left(R^{n} ; E\right)$ denotes the $E$-valued solenoidal space, i.e., closure of $\left(C_{0 \sigma}^{\infty}\left(R^{n} ; E\right)\right)^{n}$ in $\left(L^{q}\left(R^{n} ; E\right)\right)^{n}$, where

$$
C_{0 \sigma}^{\infty}\left(R^{n} ; E\right)=\left\{u \in C_{0}^{\infty}\left(R^{n} ; E\right), \operatorname{div} u=0\right\} .
$$

Let

$$
X_{q, s}=\left(H^{q, s}\left(R^{n} ; E\right)\right)^{n}, \quad X_{q, s}(A)=\left(H^{q, s}\left(R^{n} ; E(A), E\right)\right)^{n} .
$$

Let $E$ be a Banach space. Consider the space

$$
\begin{aligned}
& Y_{q}=\left\{u \in X_{q}, \operatorname{div} u \in L^{q}\left(R^{n} ; E\right)\right\}, \\
& \|u\|_{Y_{q}}=\left(\|u\|_{X_{q}}^{q}+\|\operatorname{div} u\|_{L^{q}\left(R^{n} ; E\right)}^{q}\right)^{\frac{1}{q}} .
\end{aligned}
$$

$Y_{q}$ becomes a Banach space with this norm.

It is known that (see, e.g., Fujiwara and Morimoto [4]) the vector field $u \in\left(L^{q}\left(R^{n}\right)\right)^{n}$ has a Helmholtz decomposition. In the following theorem we generalize this result for an $E$ valued function space $X_{q}$.

Theorem 4.1 Let $E$ be a UMD space and $q \in(1, \infty)$. Then $u \in X_{q}$ has a Helmholtz decomposition, i.e., there exists a bounded linear projection operator $P_{q}$ from $X_{q}$ onto $X_{q \sigma}$ with the null space $N\left(P_{q}\right)=\left\{\nabla \varphi \in X_{q}: \varphi \in L_{\mathrm{loc}}^{q}\left(R^{n} ; E\right)\right\}$. In particular, all $u \in X_{q}$ has the unique decomposition $u=u_{0}+\nabla \varphi$ with $u_{0} \in X_{q \sigma}, u_{0}=P_{q} u$ so that

$$
\|\nabla \varphi\|_{L^{q}(B ; E)}+\left\|u_{0}\right\|_{X_{q}} \leq C\|u\|_{X_{q}}
$$

for any open ball $B \subset R^{n}$. Moreover, $\left(X_{q \sigma}\right)^{*}=X_{q^{\prime} \sigma}, \frac{1}{q}+\frac{1}{q^{\prime}}=1$.

To prove Theorem 4.1, we need some lemmas. Consider the problem

$$
-\Delta_{\varepsilon} u+(A+\lambda) u=f(x), \quad x \in R^{n} .
$$


Lemma 4.1 Let $E$ be a UMD space, let $A$ be an R-positive operator in $E, q \in(1, \infty)$ and $-2<s<\infty$. Then, for $f \in H^{q, s}\left(R^{n} ; E\right), \lambda \in S_{\psi}$, problem (4.2) has a unique solution $u \in H^{q, 2+s}\left(R^{n} ; E(A), E\right)$ and the following uniform coercive estimate holds:

$$
\|u\|_{H_{\varepsilon}^{q, s+2}\left(R^{n} ; E(A), E\right)}+\|A u\|_{L^{q\left(R^{n} ; E\right)}}+|\lambda|\|u\|_{L^{q\left(R^{n} ; E\right)}} \leq C\|f\|_{H^{q, s}\left(R^{n} ; E\right)} .
$$

Proof By using the Fourier transform, we see that estimate (4.3) is equivalent to the following estimate:

$$
\begin{aligned}
\| F^{-1} & \left(1+\sum_{k=1}^{n} \varepsilon_{k} \xi_{k}^{2}\right)^{\frac{s+2}{2}} L^{-1}(\lambda, \varepsilon, \xi) \hat{f} \|_{L^{q}\left(R^{n} ; E\right)} \\
& +\left\|F^{-1} A L^{-1}(\lambda, \varepsilon, \xi) \hat{f}\right\|_{L^{q}\left(R^{n} ; E\right)}+|\lambda|\left\|F^{-1} L^{-1}(\lambda, \varepsilon, \xi) \hat{f}\right\|_{L^{q}\left(R^{n} ; E\right)} \\
\leq & C\left\|F^{-1}\left(1+|\xi|^{2}\right)^{\frac{s}{2}} \hat{f}\right\|_{L^{q}\left(R^{n} ; E\right)} .
\end{aligned}
$$

To prove (4.4) it is sufficient to show that the operator functions

$$
\left(1+\sum_{k=1}^{n} \varepsilon_{k} \xi_{k}^{2}\right) L^{-1}(\lambda, \varepsilon, \xi), \quad A\left(1+|\xi|^{2}\right)^{-\frac{s}{2}} L^{-1}(\lambda, \varepsilon, \xi), \quad|\lambda|\left(1+|\xi|^{2}\right)^{-\frac{s}{2}} L^{-1}(\lambda, \varepsilon, \xi)
$$

are multipliers in $L^{q}\left(R^{n} ; E\right)$ uniformly in $\lambda$ and $\varepsilon$. This fact is derived as the step in the proof of Theorem 3.2.

Now consider the system of equations

$$
-\Delta_{\varepsilon} u+A u+\lambda u=f(x), \quad x \in R^{n},
$$

where $f=\left(f_{1}(x), f_{2}(x), \ldots, f_{n}(x)\right) \in X_{q}$ and $u=\left(u_{1}(x), u_{2}(x), \ldots, u_{n}(x)\right)$ is a solution of (4.5).

We define in $X_{q, s}$ the following parameter-dependent norm:

$$
\|u\|_{X_{\varepsilon, q, s}}=\left\|F^{-1}\left(1+\sum_{k=1}^{n} \varepsilon_{k} \xi_{k}^{2}\right)^{\frac{s}{2}} F u\right\|_{X_{q}}<\infty
$$

Lemma 4.2 Let $E$ be a UMD space, let $A$ be an R-positive operator in $E,-2<s<\infty$ and $q \in(1, \infty)$. Then, for $f \in X_{q, s}, \lambda \in S_{\psi}$, problem (4.5) has a unique solution $u \in X_{q, s+2}$ and the following coercive uniform estimate holds:

$$
\|u\|_{X_{\varepsilon, q, s+2}}+\|A u\|_{X_{q}}+|\lambda|\|u\|_{X_{q}} \leq C\|f\|_{X_{q, s}} .
$$

Proof Problem (4.5) can be expressed as the following system:

$$
-\Delta_{\varepsilon} u_{j}+A u_{j}+\lambda u_{j}=f_{j}, \quad x \in R^{n}, j=1,2, \ldots, n .
$$

By Lemma 4.1 we obtain that for $f_{j} \in H^{q, s}\left(R^{n} ; E\right), \lambda \in S_{\psi}$, equation (4.7) has a unique solution $u=\left(u_{1}, u_{2}, \ldots, u_{n}\right) \in\left(X_{q, s+2}(A)\right)^{n}$ and the following uniform coercive estimate holds:

$$
\left\|u_{j}\right\|_{X_{\varepsilon, q, s+2}}+\left\|A u_{j}\right\|_{X_{q}}+|\lambda|\left\|u_{j}\right\|_{X_{q}} \leq C\left\|f_{j}\right\|_{X_{q, s}} .
$$


Hence, we get that $u=\left(u_{1}, u_{2}, \ldots, u_{n}\right)$ is a unique solution of problem (4.5) and (4.3) implies (4.6).

By reasoning as in [6, Lemma 2], we get the following lemma.

Lemma 4.3 $C^{\infty}\left(R^{n} ; E\right)$ is dense in $Y_{p}$.

Consider the problem

$$
-\Delta \varphi+A \varphi+\lambda \varphi=\operatorname{div} f(x), \quad x \in R^{n} .
$$

From Lemma 4.2 we obtain the following results.

Result 4.1 Let $E$ be a $U M D$ space, let $A$ be an $R$-positive operator in $E$ and $q \in(1, \infty)$. Then, for $f \in L^{q}\left(R^{n} ; E\right), \lambda \in S_{\psi}$, problem (4.8) has a unique solution $\varphi \in H^{q, 1}\left(R^{n} ; E(A), E\right)$ and the following coercive uniform estimate holds:

$$
\|u\|_{X_{\varepsilon, q, 1}}+\|A u\|_{X_{q}}+|\lambda|\|u\|_{X_{q}} \leq C\|\operatorname{div} f\|_{X_{q,-1}} .
$$

Consider the operator $P=P_{q}$ defined by

$$
D(P)=L^{q}\left(R^{n} ; E\right), \quad P f=f-\operatorname{grad} \varphi,
$$

where $\varphi$ is a solution of problem (4.8).

Result 4.2 Let $E$ be a $U M D$ space, let $A$ be an $R$-positive operator in $E$ and $q \in(1, \infty)$. Then $P_{q} X_{q}$ is a closed subspace of $X_{q}$.

Lemma 4.4 Let $E$ be a UMD space, let $A$ be an R-positive operator in $E$ and $q \in(1, \infty)$. Then the operator $P_{q}$ is a bounded linear operator in $X_{q}$ and $P f=f$ if $\operatorname{div} f(x)=0$.

Proof The linearity of the operator $P$ is clear by construction. Moreover, by Result 4.1 we have

$$
\|P f\|_{X_{q}} \leq\|f\|_{X_{q}}+\|\operatorname{grad} \varphi\|_{X_{q}} \leq C\|f\|_{X_{q}} .
$$

If $\operatorname{div} f(x)=0$, then by Lemma 4.2 we get that $\varphi=0$, i.e., $P f=f$.

Let $E^{*}$ denote the dual space of $E$.

Lemma 4.5 Assume that $E$ is a UMD space and $q \in(1, \infty)$. Then the conjugate of $P_{q}$ is defined as $P_{q}^{*}=P_{q^{\prime}}, \frac{1}{q}+\frac{1}{q^{\prime}}=1$ and is bounded linear in $\left(L^{q^{\prime}}\left(R^{n} ; E^{*}\right)\right)^{n}$.

Proof It is known (see, e.g., $[13,20])$ that the dual space of $L^{q}\left(R^{n} ; E\right)$ is $L^{q^{\prime}}\left(R^{n} ; E^{*}\right)$. Since $C_{0}^{\infty}\left(R^{n} ; E^{*}\right)$ is dense in $L^{q^{\prime}}\left(R^{n} ; E^{*}\right)$, we only have to show $P_{q}^{*} \varphi=P_{q^{\prime}} \varphi$ for any $\varphi \in\left(C_{0}^{\infty}\left(R^{n} ; E^{*}\right)\right)^{n}$. But this is derived by reasoning as in [4, Lemma 5]. Moreover, by Lemma 4.4 , the dual operator $P_{q}^{*}$ is bounded linear in $L^{q^{\prime}}\left(R^{n} ; E^{*}\right)$. 
Let

$$
\begin{aligned}
& G_{q}=\left\{\nabla \varphi: \varphi \in W^{q, 1}\left(R^{n} ; E(A) ; E\right)\right\}, \\
& \left(P_{q} X_{q}\right)^{\perp}=\left\{f \in\left(L^{q^{\prime}}\left(R^{n} ; E^{*}\right)\right)^{n},\langle f, v\rangle=0 \text { for any } v \in P_{q} X_{q}\right\} .
\end{aligned}
$$

From Lemmas 4.4, 4.5 we obtain the following result.

Result 4.3 Assume that $E$ is a $U M D$ space, $A$ is an $R$-positive operator in $E$ and $q \in(1, \infty)$. Then any element $f \in X_{q}$ uniquely can be expressed as the sum of elements of $P_{q} X_{q}$ and $G_{q}$.

In a similar way to Lemmas 6, 7 of [4] we obtain, respectively, the following lemmas.

Lemma 4.6 Assume $E$ is a UMD space and $q \in(1, \infty)$. Then

$$
\left(P_{q} X_{q}\right)^{\perp}=G_{q^{\prime}}, \quad \frac{1}{q}+\frac{1}{q^{\prime}}=1 .
$$

Lemma 4.7 Assume $E$ is a UMD space and $q \in(1, \infty)$. Then

$$
X_{q \sigma}^{\perp}=G_{q^{\prime}}, \quad \frac{1}{q}+\frac{1}{q^{\prime}}=1 .
$$

Now we are ready to prove Theorem 4.1.

Proof of Theorem 4.1 From Lemmas 4.6, 4.7 we get that $X_{q \sigma}=\left(P_{q} X_{q}\right)^{\perp}$. Then, by construction of $P_{q}$, we have $X_{q}=X_{q \sigma} \oplus G_{q}$. By Lemmas 4.2, 4.4, we obtain estimate (4.1). Moreover, by Result $4.2, G_{q}$ is a close subspace of $X_{q}$. It is known that the dual space of the quotient space $X_{q} / G_{q}$ is $G_{q}^{\perp}$. By the first assertion we have $X_{q} / G_{q}=X_{q \sigma}$, and by Lemma 4.7 we obtain the second assertion.

Theorem 4.2 Let E be a UMD space, let $A$ be an $R$-positive operator in $E, q \in(1, \infty)$. Then, for $f \in X_{q}, \varphi \in X_{q, 1}, \lambda \in S_{\psi}$, problem (1.8) has a unique solution $u \in X_{q, 2}$ and the uniform coercive estimate holds

$$
\sum_{k=1}^{n} \sum_{i=0}^{2} \varepsilon_{k}^{\frac{i}{2}}|\lambda|^{1-\frac{i}{2}}\left\|\frac{\partial^{i} u}{\partial x_{k}^{i}}\right\|_{X_{q}}+\|A u\|_{X_{q}}+\|\nabla \varphi\|_{X_{q}} \leq C\|f\|_{X_{q}}
$$

with $C=C(q, A)$ independent of $\varepsilon_{1}, \varepsilon_{2}, \ldots, \varepsilon_{n}, \lambda$ and $f$.

Proof By applying the operator $P_{q}$ to equation (1.8), we get problem (1.9). It is clear to see that

$$
D\left(Q_{\varepsilon q}\right)=D\left(B_{\varepsilon}\right) \cap X_{q \sigma},
$$

where $Q_{\varepsilon q}$ is the Stokes operator and $B_{\varepsilon}$ is an operator in $X_{q}$ generated by problem (4.5) for $\lambda=0$, i.e.,

$$
D\left(B_{\varepsilon}\right)=X_{q, 2}, \quad B_{\varepsilon} u=-\Delta u+A u .
$$


Then by Lemma 4.2 we obtain the assertion.

Result 4.4 From Theorem 4.2 we get that $Q_{\varepsilon}=Q_{\varepsilon q}$ is a positive operator in $X_{q}$ and it also generates a bounded holomorphic semigroup $S_{\varepsilon}(t)=\exp \left(-Q_{\varepsilon} t\right)$ for $t>0$.

In a similar way to that in [21] we show the following.

Proposition 4.1 The following estimate holds

$$
\left\|Q_{\varepsilon}^{\alpha} S_{\varepsilon}(t)\right\| \leq C t^{-\alpha}
$$

uniformly in $\varepsilon=\left(\varepsilon_{1}, \varepsilon_{2}, \ldots, \varepsilon_{n}\right)$ for $\alpha \geq 0$ and $t>0$.

Proof From Theorem 4.2 we obtain that the operator $Q_{\varepsilon}$ is uniformly positive in $X_{q}$, i.e., the following estimate holds

$$
\left\|\left(Q_{\varepsilon}+\lambda\right)^{-1}\right\| \leq M|\lambda|^{-1}
$$

for $\lambda \in S_{\psi, \varkappa}, 0<\psi<\pi$, where the constant $M$ is independent of $\lambda$ and $\varepsilon$. Hence, by using Danford integral and operator calculus (see, e.g., in [11]) we obtain the assertion.

\section{Well-posedness of the instationary parameter-dependent Stokes problem}

In this section, we show the uniform well-posedness of problem (1.1)-(1.2).

Theorem 5.1 Assume that $E$ is a UMD space, $A$ is an $R$-positive operator in $E$ and $0<$ $\varepsilon_{k} \leq 1$. Then, for $f \in L^{p}\left(0, T ; X_{q}\right)=B(p, q)$ and $a \in\left(X_{q, 2}(A), X_{q}\right)_{\frac{1}{p}, p}=G(p, q), p, q \in(1, \infty)$, there is a unique solution $(u, \nabla \varphi)$ of problem (1.1)-(1.2) and the following uniform estimate holds:

$$
\begin{aligned}
& \left\|\frac{\partial u}{\partial t}\right\|_{B(p, q)}+\sum_{k=1}^{n}\left\|\varepsilon_{k} \frac{\partial^{2} u}{\partial x_{k}^{2}}\right\|_{B(p, q)}+\|A u\|_{B(p, q)}+\|\nabla \varphi\|_{B(p, q)} \\
& \leq C\left(\|f\|_{B(p, q)}+\|a\|_{G(p, q)}\right)
\end{aligned}
$$

with $C=C(T, p, q)$ independent of $f$ and $\varepsilon$.

Proof Problem (1.1)-(1.2) can be expressed as the following parabolic problem:

$$
\frac{d u}{d t}+Q_{\varepsilon} u=f(t), \quad u(0)=a
$$

If we put $E=X_{q}$, then by Proposition 4.1 operator $Q_{\varepsilon}$ is uniformly positive and generates a bounded holomorphic semigroup in $X_{q}$ uniformly in $\varepsilon$. Moreover, by using [15, Theorem 3.1] we get that the operator $Q_{\varepsilon}$ is $R$-positive in $E$ uniformly in $\varepsilon$. Since $E$ is a $U M D$ space, in a similar way to that in [22, Theorem 4.2] we obtain that for $f \in L^{p}(0, T ; E)$ and $a \in\left(D\left(Q_{\varepsilon}\right), E\right)_{\frac{1}{p}, p}$, there is a unique solution $u \in W^{1, p}\left(0, T, D\left(Q_{\varepsilon}\right), E\right)$ of problem (5.2) such 
that the following uniform estimate holds:

$$
\left\|\frac{d u}{d t}\right\|_{L^{p}(0, T ; E)}+\left\|Q_{\varepsilon} u\right\|_{L^{p}(0, T ; E)} \leq C\left(\|f\|_{L^{p}(0, T ; E)}+\|a\|_{\left(D\left(A_{\varepsilon}\right), E\right)_{\bar{p}, p}}\right) .
$$

From estimates (4.10) and (5.3) we obtain the assertion.

Result 5.1 It should be noted that if $\varepsilon_{1}=\varepsilon_{2}=\cdots=\varepsilon_{n}=1$, then we obtain maximal regularity properties of an abstract Stokes problem without any parameters in principal part.

Remark 5.2 There are a lot of positive operators in concrete Banach spaces. Therefore, putting in (1.8) and (1.1) concrete Banach spaces instead of $E$ and concrete positive differential, pseudo differential operators, or finite, infinite matrices, etc. instead of $A$, by virtue of Theorem 4.2 and Theorem 5.1, we can obtain the maximal regularity properties of a different class of stationary and instationary Stokes problems which occur in numerous physics and engineering problems.

\section{Separability properties of anisotropic Stokes equations}

Let $\Omega \subset R^{m}$ be an open connected set with compact $C^{2 l}$-boundary $\partial \Omega$. Consider the BVP for the following anisotropic Stokes equations with parameters:

$$
\begin{aligned}
& (L+\lambda) u=\Delta_{\varepsilon} u+\nabla \varphi+\sum_{|\alpha| \leq 2 l} a_{\alpha}(y) D_{y}^{\alpha} u+\lambda u=f, \\
& \operatorname{div}_{x} u=0, \quad x \in R^{n}, y \in \Omega, \\
& B_{j} u=\sum_{|\beta| \leq l_{j}} b_{j \beta}(y) D_{y}^{\beta} u(x, y)=0, \quad y \in \partial \Omega, j=1,2, \ldots, l,
\end{aligned}
$$

where $a_{\alpha}$ and $b_{j \beta}$ are complex-valued functions,

$$
u=u(x, y)=\left(u_{1}(x, y), u_{2}(x, y), \ldots, u_{n}(x, y)\right), \quad \varphi=\varphi(x)
$$

are unknown solutions and

$$
f=f(x, y)=\left(f_{1}(x, y), f_{2}(x, y), \ldots, f_{n}(x, y)\right)
$$

is a given function;

$$
\Delta_{\varepsilon} u=\sum_{k=1}^{n} \varepsilon_{k} \frac{\partial^{2} u}{\partial x_{k}^{2}}, \quad D_{j}=-i \frac{\partial}{\partial y_{j}}, \quad y=\left(y_{1}, \ldots, y_{m}\right), \quad x=\left(x_{1}, \ldots, x_{n}\right),
$$

$\varepsilon=\left(\varepsilon_{1}, \varepsilon_{2}, \ldots, \varepsilon_{n}\right), \varepsilon_{k}$ are positive and $\lambda$ is a complex parameter.

If $G=R^{n} \times \Omega, \mathbf{p}=\left(p_{1}, p\right), L^{\mathbf{P}}(G)$ will denote the space of all $\mathbf{p}$-summable scalar-valued functions with mixed norm (see, e.g., [12, $\$ 1$, i.e., the space of all measurable functions $f$ defined on $G$, for which

$$
\|f\|_{L^{\mathbf{p}}(G)}=\left(\int_{R^{n}}\left(\int_{\Omega}|f(x, y)|^{p_{1}} d y\right)^{\frac{p}{p_{1}}} d x\right)^{\frac{1}{p}}<\infty
$$


Analogously, $W^{\mathbf{p}, 2,2 l}(G)$ denotes the anisotropic Sobolev space with a corresponding mixed norm $[12, \$ 10]$. Let $X_{\mathbf{p}}=\left(L^{\mathbf{p}}(G)\right)^{n}$. From Theorem 4.2 we obtain the following result.

Theorem 6.1 Let the following conditions be satisfied:

(1) $a_{\alpha} \in C(\bar{\Omega})$ for each $|\alpha|=2$ l and $a_{\alpha} \in\left[L^{\infty}+L^{\gamma_{k}}\right](\Omega)$ for each $|\alpha|=k<2 l$ with $r_{k} \geq q$ and $2 l-k>\frac{m}{r_{k}}$

(2) $b_{j \beta} \in C^{2 l-l_{j}}(\partial \Omega)$ for each $j, \beta$ and $l_{j}<2 l, \sum_{j=1}^{l} b_{j \beta}\left(y^{\prime}\right) \sigma_{j} \neq 0$, for $|\beta|=l_{j}, y^{\prime} \in \partial G$, where $\sigma=\left(\sigma_{1}, \sigma_{2}, \ldots, \sigma_{m}\right) \in R^{m}$ is normal to $\partial \Omega$;

(3) for $y \in \bar{\Omega}, \xi \in R^{m}, v \in S(\varphi), \varphi \in(0, \pi),|\xi|+|v| \neq 0$ let $v+\sum_{|\alpha|=2 l} a_{\alpha}(y) \xi^{\alpha} \neq 0$;

(4) for each $y_{0} \in \partial \Omega$, the local BVP in local coordinates corresponding to $y_{0}$

$$
\begin{aligned}
& v+\sum_{|\alpha|=2 l} a_{\alpha}\left(y_{0}\right) D^{\alpha} \vartheta(y)=0, \\
& B_{j 0} \vartheta=\sum_{|\beta|=l_{j}} b_{j \beta}\left(y_{0}\right) D^{\beta} u(y)=h_{j}, \quad j=1,2, \ldots, l,
\end{aligned}
$$

has a unique solution $\vartheta \in C_{0}\left(R_{+}\right)$for all $h=\left(h_{1}, h_{2}, \ldots, h_{m}\right) \in R^{m}$, and for $\xi^{\prime} \in R^{m-1}$ with $\left|\xi^{\prime}\right|+|\nu| \neq 0$. Then for $f \in X_{\mathbf{p}}, \lambda \in S(\varphi)$ with sufficiently large $|\lambda|$ problem (6.1)-(6.3) has a unique solution $u$ belonging to $W^{\mathbf{p}, 2,2 l}\left(G ; R^{n}\right)$ and the uniform coercive estimate holds

$$
\sum_{k=1}^{n} \sum_{i=0}^{2} \varepsilon_{k}^{\frac{i}{2}}|\lambda|^{1-\frac{i}{2}}\left\|\frac{\partial^{i} u}{\partial x_{k}^{i}}\right\|_{X_{\mathbf{p}}}+\sum_{|\beta|=2 m}\left\|D_{y}^{\beta} u\right\|_{L^{\mathbf{p}}(G)}+\|\nabla \varphi\|_{X_{q}} \leq C\|f\|_{X_{\mathbf{p}}} .
$$

Proof Let $E=L^{p_{1}}(\Omega)$. By virtue of [11, Theorem 3.6], $E$ is a $U M D$ space. Consider the operator $A$ in $L^{p_{1}}(\Omega)$ defined by

$$
D(A)=W^{p_{1}, 2 l}\left(\Omega ; B_{j} u=0\right), \quad A u=\sum_{|\alpha| \leq 2 l} a_{\alpha}(y) D^{\alpha} u(y) .
$$

Problem (6.1)-(6.3) can be rewritten in the form (1.8), where $u(x, y)=u(x, \cdot), f(x, y)=$ $f(x, \cdot)$ are vector-functions with values in $E=L^{p_{1}}(\Omega)$. By virtue of [11, Theorem 8.2] the problem

$$
\begin{aligned}
& v u(y)+\sum_{|\alpha| \leq 2 l} a_{\alpha}(y) D_{y}^{\alpha} u(y)=f(y), \\
& B_{j} u=\sum_{|\beta| \leq l_{j}} b_{j \beta}(y) D_{y}^{\beta} u(y)=0, \quad j=1,2, \ldots, l
\end{aligned}
$$

has a unique solution for $f \in L^{p_{1}}(\Omega)$ and for $v \in S(\varphi),|\nu| \rightarrow \infty$. Moreover, the operator $A$ is $R$-positive in $L^{p_{1}}(\Omega)$, i.e., all the conditions of Theorem 4.2 hold. So, we obtain the assertion. 


\section{Infinite system of Stokes equations with small parameters}

Consider the IVB for the following infinite system of instationary Stokes equations with small parameters:

$$
\begin{aligned}
& \frac{\partial u_{m}}{\partial t}-\sum_{k=1}^{n} \varepsilon_{k} \frac{\partial^{2} u_{m}}{\partial x_{k}^{2}}+\sum_{j=1}^{\infty} g_{j} u_{j}+\nabla \varphi_{m}=f_{m}(x, t), \\
& \operatorname{div} u=0, \quad u_{m}(x, 0)=0, \quad x \in R^{n}, t \in(0, T), m=1,2, \ldots, \infty,
\end{aligned}
$$

where $\varepsilon_{k}$ are positive parameters. Here $u_{m}=\left(u_{m 1}(x, t), u_{m 2}(x, t), \ldots, u_{m n}(x, t)\right), \varphi_{m}=\varphi_{m}(x, t)$ are unknown solutions, and $f=\left(f_{m 1}(x, t), f_{m 2}(x, t), \ldots, f_{m n}(x, t)\right)$ is a given function. Let

$$
\begin{aligned}
& G=\left\{g_{m}\right\}, \quad g_{m}>0, \quad u=\left\{u_{m}\right\}, \quad G u=\left\{g_{m} u_{m}\right\}, \quad m=1,2, \ldots, \\
& l_{q}(G)=\left\{u: u \in l_{\sigma},\|u\|_{l_{\sigma}(G)}=\|G u\|_{l_{\sigma}}=\left(\sum_{m=1}^{\infty}\left|g_{m} u_{m}\right|^{\sigma}\right)^{\frac{1}{\sigma}}<\infty\right\}, \quad 1<\sigma<\infty . \\
& X_{p, q, \sigma}=L^{p}\left(0, T ; L^{q}\left(R^{n} ; l_{\sigma}\right)\right) \text { is a class of functions } \\
& f=\left(f_{1}(x, t), f_{2}(x, t), \ldots, f_{n}(x, t)\right)
\end{aligned}
$$

with the norm

$$
\|f\|_{X_{p, q, \sigma}}=\left(\sum_{i=1}^{\infty}\left\|f_{i}\right\|_{L^{p}\left(0, T ; L^{q}\left(R^{n}\right)\right)}^{\sigma}\right)^{\frac{1}{\sigma}}<\infty
$$

Let $X_{p, q, \sigma}^{2}=W^{p, 2}\left(0, T ; L^{q}\left(R^{n} ; l_{\sigma}\right)\right)$. From Theorem 5.1 we obtain the following.

Theorem 7.1 Let $0<\varepsilon_{k} \leq 1$ and $g_{j}>0$. Then for $f \in X_{p, q, \sigma}, p, q, \sigma \in(1, \infty)$ there is a unique solution $\left(u_{m}, \nabla \varphi_{m}\right)$ of problem (7.1) belonging to $X_{p, q, \sigma}^{2} \times X_{p, q, \sigma}^{2}$ and the following uniform coercive estimate holds:

$$
\left\|\frac{\partial u}{\partial t}\right\|_{X_{p, q, \sigma}}+\sum_{k=1}^{n}\left\|\varepsilon_{k} \frac{\partial^{2} u}{\partial x_{k}^{2}}\right\|_{X_{p, q, \sigma}}+\|G u\|_{X_{p, q, \sigma}}+\|\nabla \varphi\|_{X_{p, q, \sigma}} \leq C\|f\|_{X_{p, q, \sigma}}
$$

with $C=C(T, p, q)$ independent of $f$ and $\varepsilon$.

Proof Really, let $E=l_{\sigma}, A$ and be an infinite matrix, defined by

$$
A=\left[g_{m} \delta_{j m}\right], \quad m, j=1,2, \ldots, \infty .
$$

It is easy to see that

$$
B(\lambda)=\lambda(A+\lambda)^{-1}=\left[\lambda\left(g_{m}+\lambda\right)^{-1} \delta_{j m}\right], \quad m, j=1,2, \ldots, \infty .
$$


For all $u_{1}, u_{2}, \ldots, u_{\mu} \in l_{q}, \lambda_{1}, \lambda_{2}, \ldots, \lambda_{\mu} \in \mathbb{C}, \lambda_{i} \neq-g_{m}, m=1,2, \ldots, \infty$ and independent symmetric $\{-1,1\}$-valued random variables $r_{i}(y), j=1,2, \ldots, \mu, \mu \in \mathbb{N}$, we have

$$
\begin{aligned}
\int_{\Omega}\left\|\sum_{i=1}^{\mu} r_{i}(y) B\left(\lambda_{i}\right) u_{i}\right\|_{l_{\sigma}}^{\sigma} d y & \leq C \int_{\Omega} \sum_{m=1}^{\infty}\left|\sum_{i=1}^{\mu} \lambda_{i}\left(g_{m}+\lambda_{i}\right)^{-1} r_{i}(y) u_{i}\right|^{\sigma} d y \\
& \leq \sup _{m, i}\left|\lambda_{i}\left(g_{m}+\lambda_{i}\right)^{-1}\right|^{\sigma} \int_{\Omega} \sum_{m=1}^{\infty}\left|\sum_{i=1}^{\mu} r_{i}(y) u_{i}\right|^{\sigma} d y .
\end{aligned}
$$

Since $\sup _{m, i}\left|\lambda_{i}\left(g_{m}+\lambda_{i}\right)^{-1}\right|^{\sigma} \leq C$ for $\lambda_{i} \neq-g_{m}$, from the above we get

$$
\int_{\Omega}\left\|\sum_{i=1}^{\mu} r_{i}(y) B\left(\lambda_{i}\right) u_{i}\right\|_{l_{\sigma}}^{\sigma} d y \leq C \int_{\Omega}\left\|\sum_{i=1}^{\mu} r_{i}(y) u_{i}\right\|_{l_{\sigma}}^{\sigma},
$$

i.e., the operator $A$ is $R$-positive in $l_{\sigma}$. Therefore, all the conditions of Theorem 5.1 hold and we obtain the assertion.

\section{Competing interests}

The author declares that they have no competing interests.

\section{Authors' contributions}

All results belong to VS.

\section{Received: 8 March 2013 Accepted: 28 June 2013 Published: 23 July 2013}

\section{References}

1. Amann, $\mathrm{H}$ : On the strong solvability of the Navier-Stokes equations. J. Math. Fluid Mech. 2, 16-98 (2000)

2. Desch, W, Hieber, M, Prüss, J: $L_{p}$-theory of the Stokes equation in a half-space. J. Evol. Equ. 1, 115-142 (2001)

3. Giga, $Y$, Sohr, H: Abstract $L_{p}$ estimates for the Cauchy problem with applications to the Navier-Stokes equations in exterior domains. J. Funct. Anal. 102, 72-94 (1991)

4. Fujiwara, D, Morimoto, $H$ : An $L_{r}$-theorem of the Helmholtz decomposition of vector fields. J. Fac. Sci., Univ. Tokyo, Sect. 1A, Math. 24, 685-700 (1977)

5. Fujita, H, Kato, T: On the Navier-Stokes initial value problem. I. Arch. Ration. Mech. Anal. 16, 269-315 (1964)

6. Farwing, R, Sohr, H: Generalized resolvent estimates for the Stokes system in bounded and unbounded domains. J. Math. Soc. Jpn. 46(4), 607-643 (1994)

7. Ladyzhenskaya, OA: The Mathematical Theory of Viscous Incompressible Flow. Gordon \& Breach, New York (1969)

8. Solonnikov, V: Estimates for solutions of nonstationary Navier-Stokes equations. J. Sov. Math. 8, 467-529 (1977)

9. Sobolevskii, PE: Study of Navier-Stokes equations by the methods of the theory of parabolic equations in Banach spaces. Sov. Math. Dokl. 5, 720-723 (1964)

10. Teman, R: Navier-Stokes Equations. North-Holland, Amsterdam (1984)

11. Denk, R, Hieber, M, Pruss, J: R-Boundedness, Fourier Multipliers and Problems of Elliptic and Parabolic Type. Mem. Amer. Math. Soc., vol. 166(788) (2003)

12. Triebel, H: Interpolation Theory. Function Spaces. Differential Operators. North-Holland, Amsterdam (1978)

13. Amann, H: Linear and Quasi-Linear Equations, vol. 1. Birkhäuser, Basel (1995)

14. Lunardi, A: Analytic Semigroups and Optimal Regularity in Parabolic Problems. Birkhäuser, Basel (2003)

15. Shakhmurov, VB: Linear and nonlinear abstract equations with parameters. Nonlinear Anal., Real World Appl. 73 , 2383-2397 (2010)

16. Shakhmurov, VB, Shahmurova, A: Nonlinear abstract boundary value problems atmospheric dispersion of pollutants. Nonlinear Anal., Real World Appl. 11(2), 932-951 (2010)

17. Shakhmurov, VB: Embedding theorems and maximal regular differential operator equations in Banach-valued function spaces. J. Inequal. Appl. 4, 605-620 (2005)

18. Shakhmurov, VB: Separable anisotropic differential operators and applications. J. Math. Anal. Appl. 327(2), $1182-1201$ (2006)

19. Yakubov, S, Yakubov, Y: Differential-Operator Equations. Ordinary and Partial Differential Equations. Chapman and Hall/CRC, Boca Raton (2000)

20. Triebel, H: Fractals and Spectra, Related to Fourier Analysis and Function Spaces. Birkhäuser, Basel (1997)

21. Kato, $T$ : Strong $L_{p}$-solutions of the Navier-Stokes equation in $R^{m}$, with applications to weak solutions. Math. Z. 187, 471-480 (1984)

22. Weis, L: Operator-valued Fourier multiplier theorems and maximal $L_{p}$ regularity. Math. Ann. 319, 735-758 (2001) 
doi:10.1186/1687-2770-2013-172

Cite this article as: Shakhmurov: Parameter-dependent Stokes problems in vector-valued spaces and applications. Boundary Value Problems 2013 2013:172.

Submit your manuscript to a SpringerOpen ${ }^{\circ}$ journal and benefit from:

- Convenient online submission

- Rigorous peer review

- Immediate publication on acceptance

- Open access: articles freely available online

- High visibility within the field

- Retaining the copyright to your article

Submit your next manuscript at $\gg$ springeropen.com 